See discussions, stats, and author profiles for this publication at: https://www.researchgate.net/publication/260843250

\title{
Staggered Grid Computation of Fluid Flow with an Improved Discretisation of Finite Differencing
}

Conference Paper in Communications in Computer and Information Science · November 2011 DOI: 10.1007/978-3-642-25462-8_8

CITATIONS

4 authors, including:

Nursalasawati Rusli

Universiti Malaysia Perlis

18 PUBLICATIONS 22 CITATIONS

SEE PROFILE
124

Ahmad Kueh

University Malaysia Sarawak

85 PUBLICATIONS 293 CITATIONS

SEE PROFILE

Some of the authors of this publication are also working on these related projects:

Project Shape Preserving Interpolation View project

Project Fluid flow and engineering problems View project 


\title{
Staggered Grid Computation of Fluid Flow with an Improved Discretisation of Finite Differencing
}

\author{
Nursalasawati Rusli ${ }^{1,2}$, Ahmad Beng Hong Kueh², and Erwan Hafizi Kasiman ${ }^{2}$ \\ ${ }^{1}$ Institute of Engineering Mathematics, Universiti Malaysia Perlis, \\ 02000 Kuala Perlis, Perlis, Malaysia \\ ${ }^{2}$ Steel Technology Centre, Faculty of Civil Engineering, \\ Universiti Teknologi Malaysia, 81310 Skudai, Johor, Malaysia \\ nursalasawati@unimap.edu.my, \{kbhahmad, erwanhafizi\}@utm.my
}

\begin{abstract}
The present paper models the fundamental problems of fluid flow using a discretely improved finite difference method on a staggered computational grid. The developed finite difference formulation is applied to well-established benchmark problems, namely, the lid-driven cavity flow, the developing laminar flow in a straight rectangular duct and the backward-facing step flow. Excellent agreements have been found for all cases. Also, this approach has successfully handled the pressure of the flow that has been long considered as one of the main problems in using the finite difference method.
\end{abstract}

Keywords: finite difference method, Navier-Stokes equations, incompressible flow, staggered grid.

\section{Introduction}

Over the past few decades, numerical modelling of fluid flow has been a major topic of research in modern science and engineering [1]. Computational fluid dynamics (CFD) occupies one of the key physical disciplines that involve the description of fluid flow in terms of mathematical models which comprise convective and diffusive transports of matters. Basically, it constitutes the groundwork covering the fields of mechanical engineering, marine engineering, aeronautics and astronautics, civil engineering and bioengineering, to name a few. Inherent in the core of fluid flow study are the mathematical models that consist of a set of governing equations in the form of ordinary or partial differential equations. Although a great account of analytical solutions for CFD is available, in practical applications, it is customary to resolve the solutions in numerical form. One of the chief techniques frequently used in the investigation of CFD is the finite difference method (FDM).

In obtaining solutions for CFD problems, one of the main concerns of the FDM is the handling of the pressure of the flow. In general, physical specification of pressure is absent, as it is implicitly correlated to the problem description. Even though there are three equations for the three unknowns $u, v, p$, there is no explicit equation which can be used for pressure. In most finite difference solution schemes for incompressible steady flows, the pressure field is obtained from a Poisson equation which is derived 